\title{
Acute correction and intramedullary nailing of aseptic oligotrophic and atrophic tibial nonunions with deformity
}

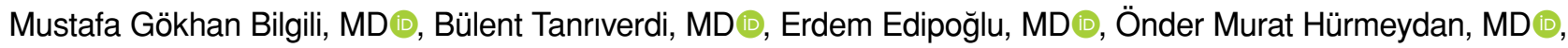

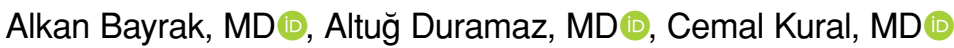

Department of Orthopedics and Traumatology, Bakırköy Dr. Sadi Konuk Training and Research Hospital, Istanbul, Turkey

Nonunion and delayed union of tibial fractures are relatively common problems and the incidence of problematic union is higher than any other long bone fractures. ${ }^{[1,2]}$ The nonunion incidence is directly related to the type of injury, trauma energy during the accident, insufficient blood supply, comminution, segmental bone loss, and soft tissue damage. ${ }^{[3]}$ Wide surgical exposure and fracture distraction might also cause nonunion. ${ }^{[4]}$

Biologically inactive nonunions are characterized with insufficient callus formation. This type of nonunions requires biological stimulation and absolute mechanical stability. ${ }^{[5]}$ Various surgical techniques such as augmentative plating, compression plate, intramedullary nailing (IMN), and external fixator can be used for nonunion treatment. ${ }^{[6]}$

Reamed IMN is a preferred treatment by many surgeons for the tibial nonunion. ${ }^{[5,6]}$ This technique provides stable fixation, correction of deformities,

Received: April 07, 2020

Accepted: May 15, 2020

Published online: September 11, 2020

Correspondence: Mustafa Gökhan Bilgili, MD. Bakırköy Dr. Sadi Konuk Eğitim ve Araştırma Hastanesi Ortopedi ve Travmatoloji Kliniği, 34147 Bakırköy, İstanbul, Türkiye.

E-mail: mgbilgili@yahoo.com

Doi: $10.5606 /$ ehc. 2020.75293

Citation: Bilgili MG, Tanrıverdi B, Edipoğlu E, Hürmeydan ÖM, Bayrak A, Duramaz A, et al. Acute correction and intramedullary nailing of aseptic oligotrophic and atrophic tibial nonunions with deformity. Jt Dis Relat Surg 2020;31(3):480-487.

(C)2020 All right reserved by the Turkish Joint Diseases Foundation

This is an open access article under the terms of the Creative Commons Attribution-NonCommercial License, which permits use, distribution and reproduction in any medium, provided the original work is properly cited and is not used for commercial purposes (http://creativecommons.org/licenses/by-nc/4.0/).

\section{ABSTRACT}

Objectives: This study aims to describe the important points for treatment of aseptic tibial oligotrophic and atrophic nonunions by intramedullary nailing (IMN).

Patients and methods: The retrospective study included 17 biologically nonactive nonunion patients (12 males, 5 females; mean age 36.4 years; range, 19 to 49 years) operated between February 2010 and November 2017 by deformity correction, static IMN and autografting. The mean follow-up time was 4.2 (range, 3 to 7 ) years. The initial fracture management was external fixator for all patients. Fourteen patients had open fractures initially. Six patients had valgus, four patients had varus, three patients had oblique plane, and four patients had external rotational deformity. Nonunion diagnosis was established on the basis of the patient history and physical examination based on plain radiographs, computed tomography or both. All patients were evaluated by the same protocol to exclude any infection.

Results: The median time from injury to nailing was mean 10.3 (range, 6.1 to 36.5 ) months. Radiologic and clinical union was achieved in all patients. The mean union time was 3.64 (range, 3 to 6) months. Three patients had positive intraoperative bacteriological culture. In four patients, dynamization was necessary for consolidation. Late deep infection developed in three patients after union, and all infected cases were operated by implant removal, debridement, and appropriate antibiotics.

Conclusion: Intramedullary nailing and autografting after external fixator provide good results for the treatment of aseptic biologically nonactive nonunions with deformity. Reamed IMN ensures sufficient deformity correction, biological environment, and mechanical stability. The infection risk should always be kept in mind and patients should be followed-up closely to prevent complications.

Keywords: Autologous bone grafting, biologically inactive nonunion, infection, intramedullary nailing.

intramedullary autografting, compression of the nonunion site, and early weight bearing. The success rates are reported as 94 to $100 \%$ in the literature. $^{[7]}$ Therefore, in this study, we aimed to describe the important points for treatment of aseptic tibial oligotrophic and atrophic nonunions by IMN. 


\section{PATIENTS AND METHODS}

This retrospective study was conducted at Orthopedics and Traumatology Clinics of Health Sciences University Bakırköy Dr. Sadi Konuk Research and Training Hospital. Seventeen nonunion patients (12 males, 5 females; mean age 36.4 years; range, 19 to 49 years) had high-energy or open tibia fracture treated with external fixator before. These patients had biologically nonactive aseptic nonunions (six oligotrophic, 11 atrophic) operated by statically locked reamed IMN between February 2010 and November 2017. Fractures were classified according to Arbeitsgemeinschaft für Osteosynthesefragen (AO) classification. All open fractures were classified according to Gustilo-Anderson classification. ${ }^{[8]}$ The study protocol was approved by the Health Sciences University, Bakırköy Dr. Sadi Konuk Training and Research Hospital Ethics Committee (Approval Date and No: 2017/59). A written informed consent was obtained from each patient. The study was conducted in accordance with the principles of the Declaration of Helsinki.

Tibial nonunion was diagnosed as a fracture that had no clinical or radiographic signs of progression to healing and very little possibility of healing without further intervention. ${ }^{[9]}$ Diagnosis was established on the basis of the patient history and physical examination (pathologic movement, pain, and inability to weight bearing). Nonunion was confirmed at the time of our initial examination on plain radiographs or by computed tomography or both. No patient in the present study had clinical or laboratory evidence of infection or any history of infection in the involved extremity. Weber and Cech classification system was used for classification of nonunion. ${ }^{[10]}$ In 10 patients who had external fixator during admission and nonunion diagnosis, fixator was removed (the lower limb was immobilized in a long-leg plaster splint) and IMN was planned after all wounds healed and laboratory tests were in normal limits. The remaining seven patients had patellar tendon bearing (PTB) brace during their admission to our clinic. All patients were treated by statically locked reamed IMN. In 12 of them, partial fibulectomy was added to the initial procedure. In five patients, fibulectomy was not necessary due to existing defect in fibula. In all patients, iliac crest autografting and backslapping technique was added to the nailing. X-ray images were evaluated according to the radiographic union scale in tibial (RUST) fractures criteria. ${ }^{[11]}$

After being discharged from hospital, the patients attended routine follow-up examinations on an outpatient basis weekly for the first month and then at monthly intervals until bony union was achieved. Each follow-up assessment included a physical examination (evaluation of mobility in the knee and ankle joints, vascular and neurological status of the limb) and an X-ray examination (anteroposterior and lateral radiographs of the tibia). The diagnosis of bone union was based on the results of the physical examination (no pain at the nonunion site and full weight-bearing capability) and posteroanterior and lateral radiographs. Radiological bone union was defined as the presence of at least three bone bridges in two projections or disappearance of the nonunion slit.

All patients were operated in supine position with radiolucent table. Fibular osteotomy and $2 \mathrm{~cm}$ fibular resection were added at the beginning of the procedure via a separate incision in 12 patients. Remaining five patients already had a defect in fibula. All patients were operated by the same surgeon. In all patients, patellar tendon splitting approach was used. Closed intramedullary technique was attempted in all patients, but in nine patients open reduction was necessary for removal of the fibrous tissue, preparing the obliterated intramedullary canal, correction of existing deformity, and proper guide wire placement. Reaming of the tibia was performed in $0.5 \mathrm{~mm}$ increments until the chattering sound was noticed and appropriate largest intramedullary nail was implanted. Intraoperative immediate Gram staining was used for detection of subtile infection. Besides, sampling from the nail surface and medullary content were used for detection of bacteria. Autografting was

\begin{tabular}{|c|c|c|c|c|}
\hline \multicolumn{5}{|c|}{$\begin{array}{l}\text { TABLE I } \\
\text { Demographic information of patients }\end{array}$} \\
\hline & \multicolumn{2}{|c|}{ Female $(n=5)$} & \multicolumn{2}{|c|}{ Male $(n=12)$} \\
\hline & Median & Min-Max & Median & Min-Max \\
\hline Mean age (year) & 35.4 & $27-44$ & 37.3 & $19-49$ \\
\hline Follow-up time (year) & 3.8 & $3-6$ & 4.6 & $3-7$ \\
\hline
\end{tabular}




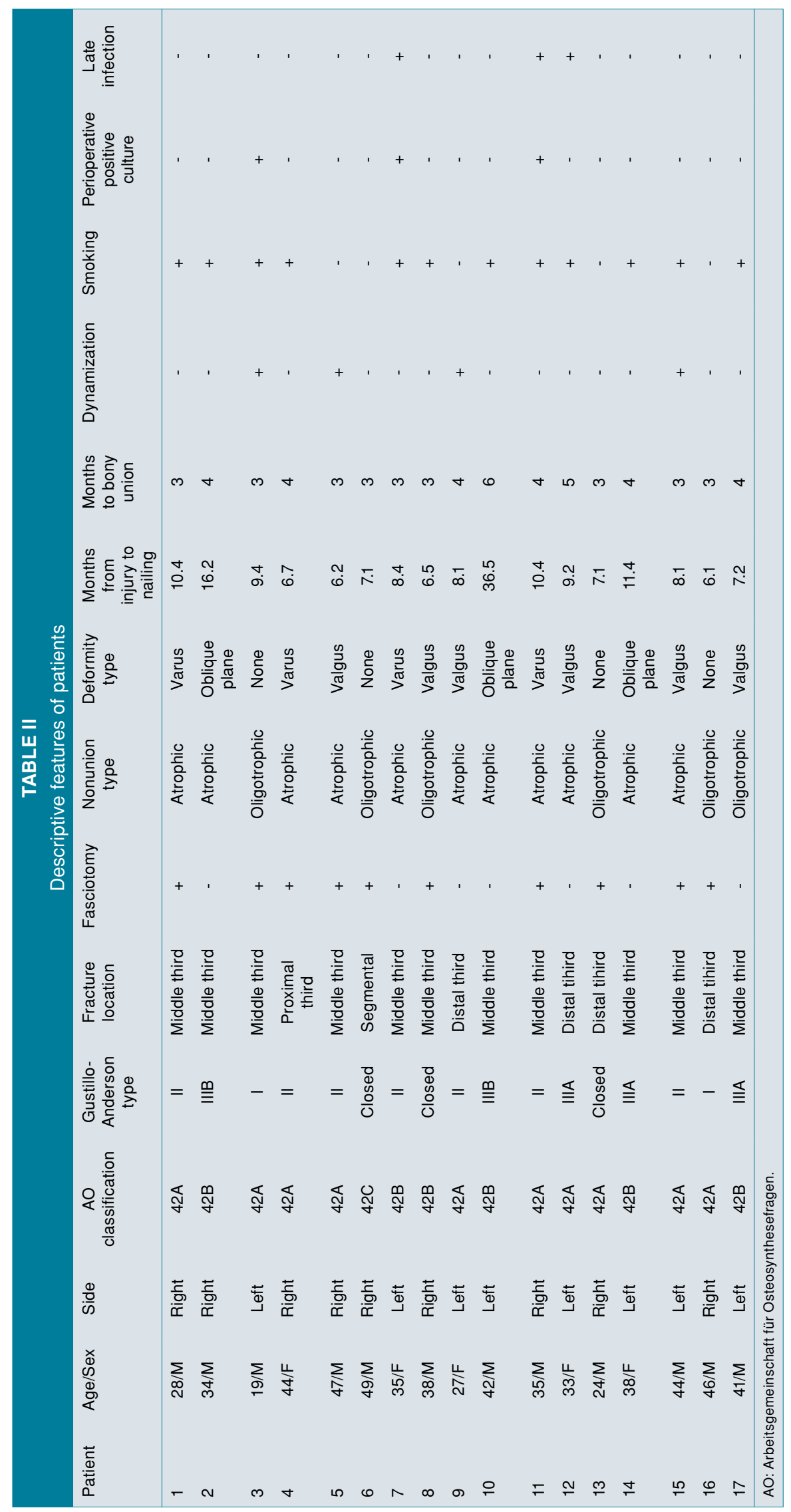


added to the procedure into the nonunion site and backslapping technique was used in all patients after distal locking to enhance bony contact. The nails were statically locked distally and proximally with two screws. Immediate partial weight bearing was allowed in all patients,

\section{Statistical analysis}

Statistical analysis was performed using the IBM SPSS version 21.0 software (IBM Corp., Armonk, NY, USA). Descriptive statistics were presented as mean \pm standard deviation, median (minimum-maximum), and $\mathrm{n}(\%)$ values, where appropriate. Fisher's exact test and Pearson's chi-square analysis were performed for categorical variables. Mann-Whitney $\mathrm{U}$ test was used for comparison of quantitative variables with non normal distribution. A $p$ value $<0.05$ was considered statistically significant.

\section{RESULTS}

Demographical data and information on primary injuries, all previous treatments, and associated diseases were obtained from medical records (Table I). Among 17 patients, the right tibia was fractured in nine patients and the left in eight. The mean follow-up time was 4.2 (range, 3 to 7 ) years (Table II). High-energy trauma accounted for all initial fractures. The location of the fracture was the proximal third in one patient, middle third in 11 patients, and distal third in four patients while one fracture was originally segmental.

According to $\mathrm{AO}$ classification, 10 patients had $42 \mathrm{~A}$, six patients had $42 \mathrm{~B}$, and one patient had $42 \mathrm{C}$ fracture. Fourteen patients had open fractures initially (two grade I, seven grade II, three grade IIIA, and two grade IIIB). Six patients had valgus deformity, four patients had varus deformity, and three patients had oblique plane deformity. In four patients, there was external rotational deformity. All patients were previously treated by external fixator. In 10 patients, compartment syndrome was diagnosed during initial trauma and treated by fasciotomy.
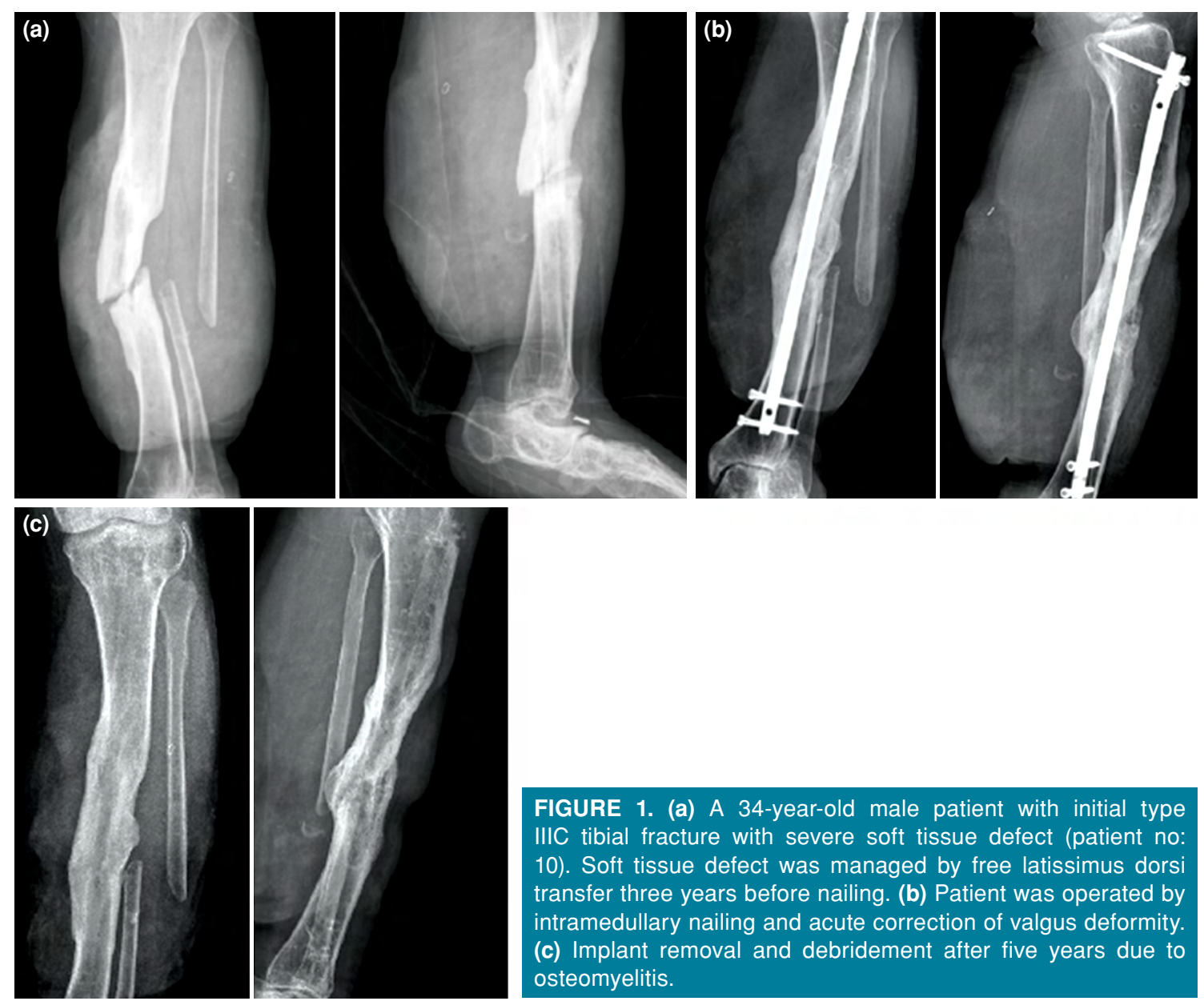

FIGURE 1. (a) A 34-year-old male patient with initial type IIIC tibial fracture with severe soft tissue defect (patient no: 10). Soft tissue defect was managed by free latissimus dorsi transfer three years before nailing. (b) Patient was operated by intramedullary nailing and acute correction of valgus deformity. (c) Implant removal and debridement after five years due to osteomyelitis. 
The mean time from injury to nailing averaged 10.3 (range, 6.1 to 36.5) months. Radiologic and clinical union was achieved in all patients. The mean union time was 3.64 (range, 3 to 6) months according to the RUST criteria. No statistically significant difference was observed in terms of union time between sexes $(\mathrm{p}=0.624)$ and smoking habit $(\mathrm{p}=0.612)$ (Table I). The mean PTB using time was 11 (range, 6 to 36) months. In 10 patients, the mean time from the removal of external fixator pins to the secondary IMN of the tibia was 18 days (range, 14 days to one month). Three patients had positive intraoperative bacteriological culture, but none of them developed early postoperative infection. Methicillin-sensitive Staphylococcus aureus was isolated in two patients and Staphylococcus epidermis was isolated in one patient at perioperative culture tests. However, infective nonunion was not detected in clinical and radiological evaluations in any patient with preoperative culture positivity. Therefore, those patients were considered as aseptic nonunion. In four patients, dynamization was added to the treatment. All tibial nonunions healed uneventfully while late deep infection developed after union in three patients, and all infected cases were operated by implant removal, debridement, and appropriate antibiotics. Only one of them had perioperative positive culture. Staphylococcus epidermis was the causative agent in those three late infection cases. No osteomyelitis occurred in any of the patients after the 3.6-year mean follow-up time.
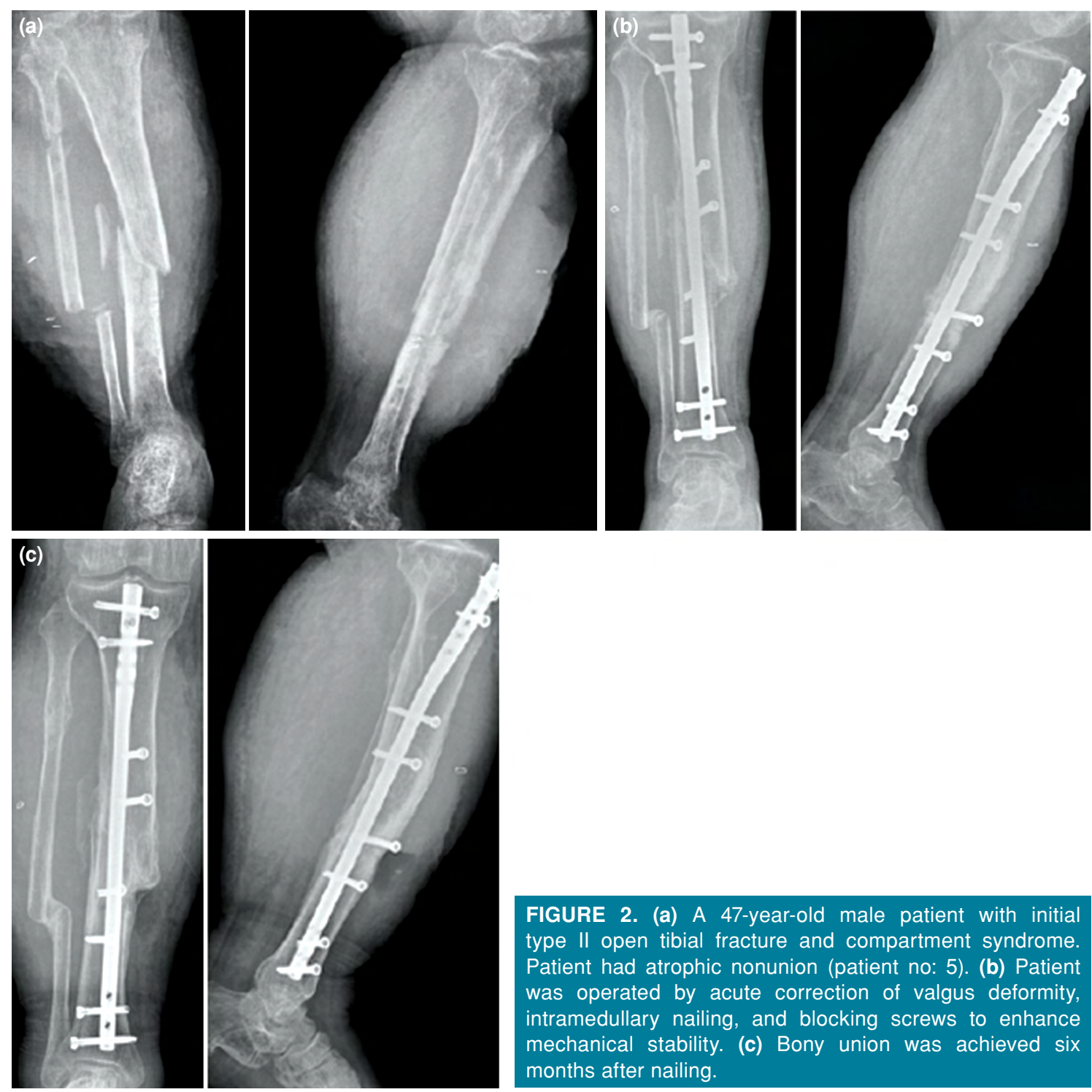

FIGURE 2. (a) A 47-year-old male patient with initial type II open tibial fracture and compartment syndrome. Patient had atrophic nonunion (patient no: 5). (b) Patient was operated by acute correction of valgus deformity, intramedullary nailing, and blocking screws to enhance mechanical stability. (c) Bony union was achieved six months after nailing. 


\section{DISCUSSION}

Different time points have been reported in the literature to define delayed union or nonunion of the tibia. Nonunion was defined as lack of healing after nine months following injury. ${ }^{[12,13]}$ Absence of progressive healing on radiographs for three months may also help to diagnose nonunion. ${ }^{[13]}$ Pain with weight bearing, pathological motion, loosening or displacement of fixation material may also help for the diagnosis. ${ }^{[14,15]}$ In the present study, diagnosis was established on the basis of the patient history and physical examination (pathologic movement, pain, and inability to weight bearing). Nonunion was defined as inability to heal without further intervention and meticulous diagnostic tests were used to exclude infection.

All tibial nonunion patients should be evaluated for septic nonunion. Erythrocyte sedimentation rate and C-reactive protein are useful laboratory tests to rule out the infection. White blood cell count may be normal in infected cases. Even in patients with normal laboratory test results, positive intraoperative bacterial culture results may occur in $28 \%$ of the patients. ${ }^{[14]}$ Intraoperative bacterial cultures should be taken from the medullary cavity and existing implant surface. Pre- and perioperative exclusion of infection is obligatory for treatment of tibial nonunions. ${ }^{[15-18]}$ In the current study, three patients had perioperative positive culture result (17\%) despite all laboratory tests with normal limits, negative peroperative Gram staining, and no signs of infection in preoperative physical examination. It is obvious that one should keep in mind subtile infection in the treatment of pseudoarthrosis.

Mechanical instability is another concern for all types of nonunions. Adequate fixation provides biomechanically stable construct, eliminates disturbing forces, and promotes healing. ${ }^{[19,20]}$ Blocking screws may be necessary to correct the deformity or enhance the mechanical stability. Osteotomy shape, site, technique, grafting of the nonunion site, appropriate nail length and thickness, reaming amount, correction degree, and lengthening should be calculated preoperatively. Valgus deformities should take major concern because of the peroneal nerve. If there is any suspicion that the correction amount may give harm to the peroneal nerve, the fibular nerve should be released before nailing, particularly in patients with valgus deformity. We prefer static locking technique in all patients to provide sufficient stability and early weight bearing for axial compression.
The medullary canal is blocked by the bony tissue in most of the tibial nonunion cases. Medullary canal should be reamerized and adequate mechanical stability should be achieved by the appropriate nail size to replace the nail properly. Open reduction of the nonunion site, retrograde reaming of the proximal segment, and antegrade reaming of the distal segment are often necessary because of the obstructed medullary canal. In this study, open reduction was necessary in nine patients for proper guide wire placement and opening of the obliterated medullary canal. This incision can also be used for correction of the deformity and autografting.

Different surgical techniques might be preferred in tibial nonunion treatment. Unfortunately, nonunion was the most prevalent complication of tibial shaft fractures due to insufficient soft tissue cover and high incidence of open fractures independent of the surgical fixation methods such as IMN, locking compression plating, or external fixation. ${ }^{[20]}$ Intramedullary nailing provides more mechanical stability, enhances biological environment by autografting due to reaming, and supplies dynamic compression in the nonunion site.

Bone contact is obligatory for the treatment of nonunions. Intramedullary reaming already provides internal grafting and biological environment for healing, while delayed union or nonunion may occur in cases with bone gap. Generally, additional interventions should be preferred in failed revision cases and in patients with severe bone loss (more than $2 \mathrm{~cm}$ or more than $50 \%$ of bone circumference). In aseptic oligotrophic/atrophic nonunions, autografting may enhance biologic environment and increase bony contact. ${ }^{[21,22]}$ Also, backslapping technique may increase the bony contact and compress the nonunion site. In the current study, autografting and backslapping were added to the IMN. In all patients, bone union was achieved with this technique. Intramedullary reaming provides sufficient biologic environment for healing and addition of grafting and backslapping may enhance the healing process.

Fibulectomy may be required for the correction of tibial deformity. Also, a healed fibula may cause delayed union or nonunion of the tibia particularly in defected cases. If the backslapping technique is preferred to enhance the bone contact, a united fibula may also prevent adequate compression on the nonunion sites. Therefore, the fibulotomy/ fibulectomy choice should be individualized and preferred accordingly. ${ }^{[15,23]}$ If the fibula is displayed with signs of bowing or remodeling, suggesting that the fibula was preventing compression at 
the nonunion site, fibulotomy should be added other than that of the nonunion site to prevent tibial destabilization. ${ }^{[24,25]}$ In the current study, fibulectomy was added to the main procedure in 12 patients, and there was already fibular defect in five patients. In atrophic or oligotrophic nonunions, routine fibulectomy may increase the backslapping effect and also provide axial compression during weight bearing. Therefore, we recommend routine fibulectomy in biologically non-active nonunions to increase bony contact, correct the deformity, and provide early axial compression.

The limitations of this study include the retrospective design, the small sample size, lack of a control group, presence of heterogeneity in terms of open fracture types, and slightly short follow-up period. However, the homogeneous patient group and the same treatment protocol by one surgeon are the powerful sides of the study. Further randomized studies with larger cohort populations including control groups are necessary to ascertain the results of this treatment protocol.

In conclusion, aseptic tibial oligotrophic or atrophic nonunion with deformity management is challenging despite a great number of treatment alternatives. A successful treatment strategy requires meticulous preoperative patient assessment, accurate identification of nonunion type, regular evaluation of soft tissue condition, and adequate equipment for the surgery. The aim of treatment should involve immediate weight bearing, increased mechanical stability, sufficient biological environment for healing, and preventing multiple surgeries and prolonged disability. IMN and autografting after external fixator provide good results for the treatment of aseptic biologically nonactive nonunions with deformity. Reamed IMN ensures sufficient deformity correction, biological environment, and mechanical stability. The infection risk should always be kept in mind and patients should be followed-up closely to prevent complications.

\section{Declaration of conflicting interests}

The authors declared no conflicts of interest with respect to the authorship and/or publication of this article.

\section{Funding}

The authors received no financial support for the research and/or authorship of this article.

\section{REFERENCES}

1. Boyd HB, Lipinski SW, Willey JH. Observations on nonunion of the shafts of long bones with a statistical analysis of 842 patients. J Bone Joint Surg 1961;43A:159.
2. Ellis $\mathrm{H}$. The speed of healing after fracture of the tibial shaft. J Bone Joint Surg [Br] 1958;40:42-6.

3. Heppenstall RB, Brighton CT, Esterhai JL Jr, Muller G. Prognostic factors in nonunion of the tibia: an evaluation of 185 cases treated with constant direct current. J Trauma 1984;24:790-5.

4. Çiçekli Ö, Kochai A, Şükür E, Başak AM, Kurtoğlu A, Türker M. Suprapatellar approach for fractures of the tibia: Does the fracture level matter? Eklem Hastalik Cerrahisi 2019;30:10-6.

5. Lambiris E, Tyllianakis M, Megas P, Panagiotopoulos E. Intramedullary nailing: experience in 427 patients. Bull Hosp Jt Dis 1996;55:25-7.

6. Lottes JO. Treatment of delayed or nonunion fractures of the tibia by a medullary nail. Clin Orthop Relat Res 1965;43:111-28.

7. Megas P, Panagiotopoulos E, Skriviliotakis S, Lambiris E. Intramedullary nailing in the treatment of aseptic tibial nonunion. Injury 2001;32:233-9.

8. Gustilo RB, Merkow RL, Templeman D. The management of open fractures. J Bone Joint Surg [Am] 1990;72:299-304.

9. Swanson EA, Garrard EC, O'Connor DP, Brinker MR. Results of a systematic approach to exchange nailing for the treatment of aseptic tibial nonunions. J Orthop Trauma 2015;29:28-35.

10. Weber B, editor. Pseudarthrosis: Pathophysiology, biomechanics, therapy, resuls. Bern Switzerland: Hans Huber; 1976.

11. Leow JM, Clement ND, Tawonsawatruk T, Simpson CJ, Simpson AH. The radiographic union scale in tibial (RUST) fractures: Reliability of the outcome measure at an independent centre. Bone Joint Res 2016;5:116-21.

12. Phieffer LS, Goulet JA. Delayed unions of the tibia. J Bone Joint Surg [Am] 2006;88:206-16.

13. Goulet JA, Templeman D. Delayed union and nonunion of tibial shaft fractures. Instr Course Lect 1997;46:281-91.

14. Bhattacharyya T, Bouchard KA, Phadke A, Meigs JB, Kassarjian A, Salamipour H. The accuracy of computed tomography for the diagnosis of tibial nonunion. J Bone Joint Surg [Am] 2006;88:692-7.

15. Hak DJ. Management of aseptic tibial nonunion. J Am Acad Orthop Surg 2011;19:563-73.

16. Amorosa LF, Buirs LD, Bexkens R, Wellman DS, Kloen $\mathrm{P}$, Lorich DG, et al. A single-stage treatment protocol for presumptive aseptic diaphyseal nonunions: a review of outcomes. J Orthop Trauma 2013;27:582-6.

17. Kostic I, Mitkovic M, Mitkovic M. The diaphyseal aseptic tibial nonunions after failed previous treatment options managed with the reamed intramedullary locking nail. J Clin Orthop Trauma 2019;10:182-90.

18. Arsoy D, Donders JCE, Kleeblad LJ, Miller AO, Henry MW, Wellman DS, et al. Outcomes of presumed aseptic long-bone nonunions with positive intraoperative cultures through a single-stage surgical protocol. J Orthop Trauma 2018;32 Suppl 1:S35-S9.

19. Mast JW. Preoperative Planning in the Surgical Correction of Tibial Nonunions and Malunions. J Orthop Trauma 2018;32 Suppl 1:S1-S4.

20. Hierholzer C, Friederichs J, Glowalla C, Woltmann A, Bühren V, von Rüden C. Reamed intramedullary exchange nailing in the operative treatment of aseptic tibial shaft nonunion. Int Orthop 2017;41:1647-53. 
21. Chalidis BE, Petsatodis GE, Sachinis NC, Dimitriou CG, Christodoulou AG. Reamed interlocking intramedullary nailing for the treatment of tibial diaphyseal fractures and aseptic nonunions. Can we expect an optimum result? Strategies Trauma Limb Reconstr 2009;4:89-94.

22. Le Baron M, Vivona JP, Maman P, Volpi R, Flecher X. Can the Reamer/Irrigator/Aspirator System replace anterior iliac crest grafting when treating long bone nonunion? Orthop Traumatol Surg Res 2019;105:529-33.
23. Aldemir C, Duygun F. Outcome of locked compressive nailing in aseptic tibial diaphyseal nonunions without bone defect. Indian J Orthop 2019;53:251-6.

24. Connolly JF. Common avoidable problems in nonunions. Clin Orthop Relat Res 1985;(194):226-35.

25. Duygun F, Aldemir C. Effect of intramedullary nail compression amount on the union process of tibial shaft fracture and the evaluation of this effect with a different parameter. Eklem Hastalik Cerrahisi 2018;29:87-92. 